\title{
Low Voltage System State Estimation based on Smart Metering Infrastructure
}

\author{
Marco Pau*, Edoardo Patti ${ }^{\dagger}$, Luca Barbierato ${ }^{\dagger}$, ${\text { Abouzar Estebsari }{ }^{\ddagger}, \text { Enrico Pons }}^{\ddagger}$, \\ Ferdinanda Ponci*, Antonello Monti* \\ *Institute for Automation of Complex Power Systems, RWTH Aachen University, Aachen, Germany \\ $\dagger$ Department of Control and Computer Engineering, Politecnico di Torino, Turin, Italy \\ $\ddagger$ Department of Energy, Politecnico di Torino, Turin, Italy
}

\begin{abstract}
The accurate monitoring of distribution grids is essential to enable the intelligent management and control of future Smart Grids. Several challenges prevent an easy development of the state estimation tools needed to assess the operating conditions of distribution networks. The lack of a suitable measurement infrastructure is one of the most challenging aspects to face. However, in last years, several utilities started a massive deployment of smart meters in their networks. The proper use of these measurements is key to enhance the performance of distribution system state estimators. This paper presents a two-level approach conceived to efficiently include smart meter measurements in low voltage grid state estimation. The proposed solution relies on a cloud-based smart metering architecture, which allows scalability and interoperability among different off-the-shelf meters. Moreover, a suitable design of the estimation algorithm, using the uncertainty propagation theory, is proposed in order to maximize the accuracy of the estimation results. Tests performed on a sample low voltage network show the performance and the main features of the proposed state estimation solution.
\end{abstract}

Index Terms-State Estimation, Smart Meters, Cloud Architecture, Distribution Grids, Measurement Uncertainty.

\section{INTRODUCTION}

The accurate knowledge of the operating conditions is the basis for most of the management and control functions used to efficiently operate the electrical grids. State Estimation (SE) is the tool commonly adopted to achieve the needed situational awareness [1]. Nowadays SE algorithms are mainly used in transmission systems, but with the ongoing transition of the distribution networks towards active grids, and the perspective of the Smart Grid (SG) scenario, SE is expected to play a key role also at distribution level [2].

Different challenges hinder the efficient deployment of SE in distribution grids [3]. One of the main issues is the lack of suitable measurement infrastructures. This generally implies the necessity to use the so-called pseudo-measurements, namely forecast measurements derived from historical and/or statistical data about load consumption and power generation. The use of pseudo-measurements allows reaching the network observability, but it usually leads to poor accuracy in the SE

(c) 2016 IEEE. Personal use of this material is permitted. Permission from IEEE must be obtained for all other users. DOI: 10.1109/AMPS.2016.7602804. Publisher version: http://ieeexplore.ieee.org/ document/7602804/ results, since this information is not highly reliable and is consequently characterized by large uncertainty [4].

In last years, thanks to legislations aiming at improving the efficiency in the energy sector, many countries started the deployment of advanced smart metering infrastructures in the distribution grids. In Europe, the Commission Recommendation 2012/148/EU paved the way for the smart meter roll-out in several countries [5]. According to the report issued in 2014 by the European Commission, after a long term costs and benefits analysis, 16 European Countries decided to invest in a large roll-out of smart meters that will lead to the deployment of almost 200 million devices by 2020 [6].

The deployment of smart metering infrastructures enables the implementation of advanced services for both customers and Distribution System Operators (DSOs). To this purpose, several research projects have been recently funded aimed at developing innovative products based on the use of smart meters [7]-[9]. SE is of course one of the possible applications that could gain great benefit from the availability of this measurement infrastructure. Smart meter measurements can be in fact used to obtain reliable information on the customer power consumptions, to enlarge the measurement redundancy and to improve the SE accuracy performance.

In the literature, some papers already faced some issues related to the use of smart meters in SE. In [10] and [11] the focus is on the design of efficient smart meter based estimators and on the analysis of the impact brought by these measurements, while [12] and [13] deal with the problem of unavailable real-time communication or synchronization in the data transmission. In this paper the goal is twofold. First, a cloud-based architecture, conceived in the context of the Flexmeter project [7] for handling the smart meter measurements, is presented in Section II. The used cloud solution provides several advantages, such as: the easy plug and play implementation of new devices, the interoperability among different off-the-shelf meters, the access to specific sets of measurements for different service providers, large flexibility and scalability. In Section III, instead, a SE algorithm tailored to the adopted smart metering architecture is presented. The proposed approach relies on a first level estimation executed in distributed intelligent nodes, which permits scaling down the SE problem, followed by a second level estimation computing the final state of the LV feeders. The uncertainty propagation 


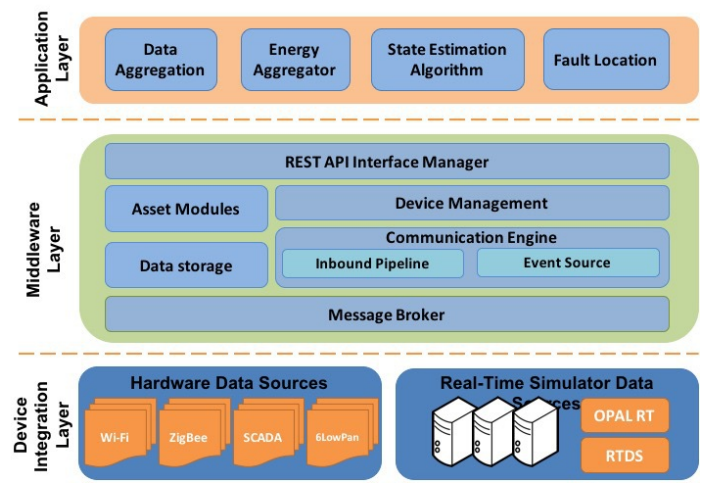

Fig. 1. Architectural schema of Flexmeter Multi Metering Platform

theory is used in the SE process to enhance the achievable accuracy, in order to get the largest benefit from the use of the smart metering infrastructure. Performance and features of the proposed SE solution are finally shown in Section IV through tests performed on a simple LV test network.

\section{Software Architecture For SMart Metering}

The main purpose of a smart metering architecture is enabling and fostering new services to make a more reliable and secure network management. The most relevant issue of these architectures concerns the interoperability between heterogeneous metering devices. Service providers need a common data access to this large amount of incoming information, abstracting from the different underlying low-level technologies. To cope with this issue, we present Flexmeter, which is a software infrastructure for smart metering. As shown in Figure 1, Flexmeter is a three layer architecture with: i) a Device Integration Layer, ii) a Middleware Layer and iii) an Application Layer. The rest of this section describes each layer of the proposed infrastructure in more detail.

\section{A. Device Integration Layer}

A Smart Metering Architecture has to deal with heterogeneous devices that exploit different communication protocols (e.g. IEEE 802.11, ZigBee, SCADA or 6LowPan). The Device Integration Layer is the lower layer of the proposed infrastructure (see Figure 1). It abstracts different hardware data sources leveraging upon entities called Device Integration Adapters (DIA) [14]. A DIA converts the measurements coming from the devices to the Flexmeter Data Format and send them to the cloud infrastructure via MQTT [15], which exploits publish/subscribe communication paradigm [16]. In addition, we developed a specific DIA to integrate Real-Time Digital Simulators, such as RTDS and Opal RT, into the Flexmeter infrastructure.

\section{B. Middleware Layer}

As shown in Figure 1, the Middleware Layer is composed by several software components acting together to: 1) allow MQTT communication with Device Integration Adapters; 2) receive, control and store measurement messages and 3) provide REST [17] APIs to access data, devices, assets and

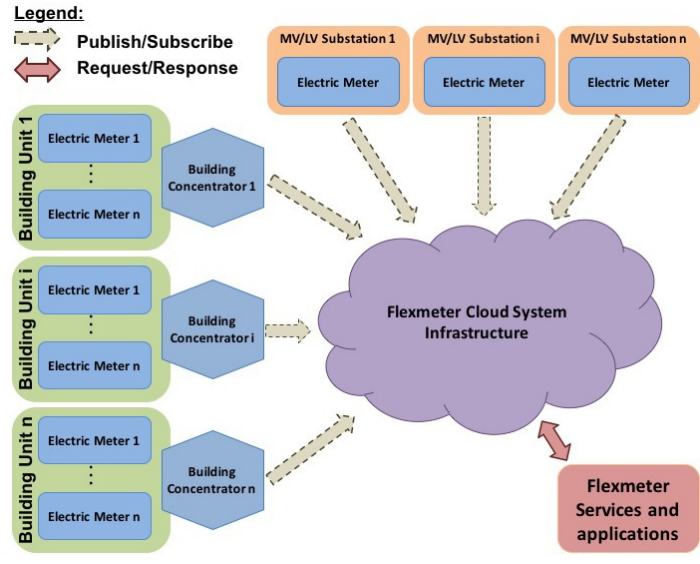

Fig. 2. Flexmeter Cloud Communication Diagram

maintenance operations. The Message Broker provides an asynchronous communication through MQTT [15]. MQTT is a publish/subscribe protocol [16] to send data in (near-)realtime. This approach decouples network traffic between information producers and consumers increasing the scalability of the whole infrastructure [18]. The Message Broker is also in charge of routing all events to the Communication Engine. In its core, the Communication Engine consists of other two components: i) Event Sources and ii) Inbound Pipeline. The Event Source receives messages from the Message Broker, checks the integrity and push them into the Inbound Pipeline. Inbound Pipeline manages message network traffic spikes to relieve the database interface from congestion and to ensure the measurements storage. Data Storage is an interface designed to integrate different non relational database models (e.g. document-oriented or time series). This approach helps in scalability and clusterization keeping independent the cloud infrastructure from the low-level database management systems. Device Management handles the interactions between devices and application layer. Asset Modules manages different information regarding people, places and things that are called assets in Flexmeter. The main asset represents physical location of electric smart meters. It contains all of the core asset management calls including CRUD methods for asset categories and assets. Finally, REST API Interface Manager provides REST API to access information and manage entities or devices in the infrastructure. All REST calls are subject to authentication to check if the user is authorized in performing operations.

\section{Application Layer}

The Application Layer offers the interface for different applications to design complex services. For instance, Data and Energy Aggregators can apply Demand Side Management and Demand Response programs on specific portions of the network. SE Algorithm can enable near real-time monitoring of the system here meaning the monitoring of the operating conditions of the grid with a delay in the order of few seconds), allowing security, reliability and efficiency oriented management of the grid. 


\section{Data and communication flows in Flexmeter}

In this section we describe the data and communication flows in Flexmeter from the devices (i.e. smart meter) to services and applications. As shown in Figure 2, the infrastructure exploits the two main communication paradigms: request/response (i.e. REST [17]) and publish/subscribe (i.e. MQTT [15]). The applications (e.g. Distribution System State Estimation algorithm) provide their functionalities through web service interfaces designed with a REST approach. In the Flexmeter scenario, off-the-shelf smart meters are installed at end user premises and they send measurements to a building concentrator. Additional MV/LV substation meters can be deployed along the electricity grid network to enhance the monitoring of the distribution system status. Both concentrators and MV/LV substation meters send data to Flexmeter Cloud System Infrastructure through MQTT. Such information is received by the cloud, which is in charge of: i) collecting and storing measurements; ii) provide REST API to permit interaction with data, devices and assets management. Finally, applications retrieve data from the platform through the REST API in order to post-process them and provide a new service for end-users or Smart Grid maintenance.

\section{StATE Estimation ALGORITHM}

\section{A. SE architecture}

As shown in Fig. 2, building concentrators play a key role in the smart metering architecture here presented. These concentrators are the intelligent nodes where the smartness of the measurement system resides. Thanks to specifically designed interfaces, they allow achieving interoperability across heterogeneous commercial devices that implement different communication protocols. In addition, they are responsible for aggregating meter readings related to different customers and for transmitting them to the central cloud system, where they are made available for the different functions and services.

From a SE perspective, the computational capabilities available in the concentrators can also be used to distribute some of the requirements associated to SE. Fig. 3 shows a schematic view of the conceived solution. As it can be observed, the typical radial configuration of LV grids is exploited to design a two-level approach. In the first level, SE is performed locally in each concentrator for the small portion of the grid associated to the customers under the considered building unit. The resulting estimates of voltage and power injection at the upstream feeder bus are then sent to the central cloud system. Subsequently, at the second stage, the central LV grid SE retrieves these estimates from the cloud and utilizes them as equivalent input measurements for obtaining the overall view of the operating conditions of the LV feeder. The proposed scheme needs a suitable placement of concentrators, but it allows achieving several benefits.

- The accurate measurements available from the end-user smart meters are fully exploited to achieve the estimation of the LV grid without the need of pseudo-measurements.

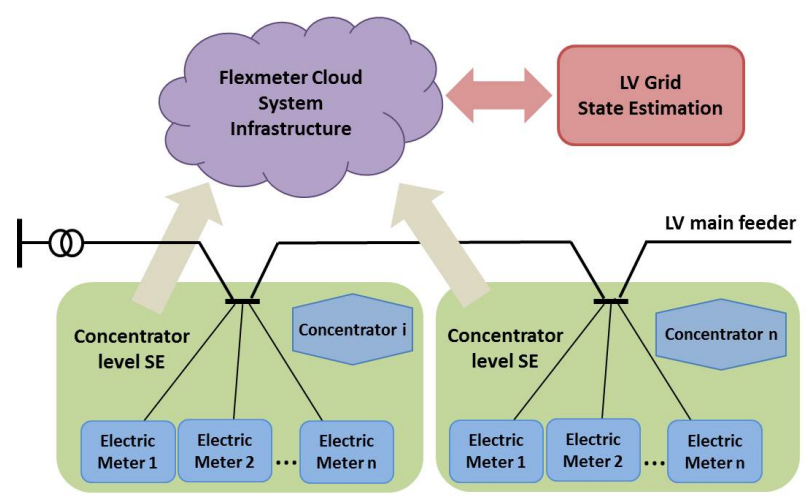

Fig. 3. Two-level SE architecture

- At the first level, SE is performed in parallel and independently in different portions of the grid, thanks to the availability of the distributed concentrators.

- The building concentrators have to send to the cloud only a small amount of data (the resulting estimates at the feeder bus), thus limiting the requirements for real-time communication.

- The central SE only uses the relevant information at the feeder buses, without the need to collect, store and process all the meter readings associated to each customer.

\section{B. SE model}

The well-known Weighted Least Squares (WLS) method is used to perform SE both at concentrator and central level. The approach refers to the following measurement model:

$$
\mathbf{y}=\mathbf{h}(\mathbf{x})+\mathbf{e}
$$

where $\mathbf{y}$ is the vector of input measurements, $\mathbf{x}$ is the chosen vector of state variables, $\mathbf{h}(\mathbf{x})$ is the set of functions expressing the measured quantities in terms of the state variables, and $\mathbf{e}$ is the error vector associated to the input measurements.

Starting from this measurement model, the WLS method aims at minimizing the following objective function:

$$
J(\mathbf{x})=[\mathbf{y}-\mathbf{h}(\mathbf{x})]^{T} \mathbf{W}[\mathbf{y}-\mathbf{h}(\mathbf{x})]
$$

where $\mathbf{W}$ is a weighting matrix equal to the inverse of the measurement error covariance matrix used to provide more weight to the measurements with more accuracy.

The minimization of the objective function in (2) is usually performed applying iteratively the Gauss-Newton method, which leads to the following equation system to be solved at each iteration of the estimation algorithm:

$$
\mathbf{G} \Delta \mathbf{x}=\mathbf{H}^{T} \mathbf{W}[\mathbf{y}-\mathbf{h}(\mathbf{x})]
$$

where $\mathbf{G}=\mathbf{H}^{T} \mathbf{W H}$ is the so-called Gain matrix, $\mathbf{H}$ is the Jacobian of the measurement functions $\mathbf{h}(\mathbf{x})$, and $\Delta \mathbf{x}$ is the vector needed to update the state variables at each iteration $k$ according to the following:

$$
\mathbf{x}_{k+1}=\mathbf{x}_{k}+\Delta \mathbf{x}
$$

In this paper, an estimator using rectangular voltages as state variables has been chosen, since this formulation is particularly 
efficient when a large number of voltage magnitude measurements has to be processed [19]. Given the unbalanced nature of the LV grids, a three-phase estimator is considered. It is worth noting that, generally, in the three-phase model of the grid, mutual impedances should be duly taken into account [20]. However, in most of the practical scenarios this information is usually not available. As a result, a phase-decoupled version of the three-phase estimator can be designed, which allows parallel execution of SE in the three phases.

In the proposed estimator, an important aspect concerns the integration between the SE processes at concentrator and central level. As mentioned above, each local estimation carried out in the concentrators provides as output the voltage magnitude and the equivalent active and reactive power injection at the connection node in the feeder. To properly implement the WLS procedure in the upper level SE, the uncertainties of these estimates have to be calculated, in order to correctly derive the needed weights. As also shown in the literature (see for example [21]), this step is essential to achieve the maximum benefit in terms of SE accuracy.

To obtain the needed uncertainties, the first step is the calculation of the state estimates covariance matrix $\boldsymbol{\Sigma}_{\mathbf{x}}$. In the WLS approach, this information is simply given by the inverse of the Gain matrix used in (3):

$$
\boldsymbol{\Sigma}_{\mathbf{x}}=\mathbf{G}^{-1}
$$

Starting from the covariance matrix of the state estimates, the uncertainty associated to the desired quantities can be obtained by applying the theory of propagation of the uncertainty by means of:

$$
\boldsymbol{\Sigma}_{v p q}=\left[\frac{\partial \mathbf{f}}{\partial \mathbf{x}}\right]\left[\boldsymbol{\Sigma}_{\mathbf{x}}\right]\left[\frac{\partial \mathbf{f}}{\partial \mathbf{x}}\right]^{T}
$$

where $\boldsymbol{\Sigma}_{v p q}$ is the covariance matrix related to the voltage and power injection estimates at the feeder bus and $\left[\frac{\partial \mathbf{f}}{\partial \mathbf{x}}\right]$ is a matrix having in the rows the partial derivatives, with respect to the state variables $\mathbf{x}$, of the following functions:

$$
\begin{gathered}
\mathbf{f}_{v}=\sqrt{v_{r, f}^{2}+v_{x, f}^{2}} \\
\mathbf{f}_{p}=\left(v_{r, f}^{2}+v_{x, f}^{2}\right) \cdot \sum_{i \in \Gamma} g_{f i}-v_{r, f} \cdot \sum_{i \in \Gamma}\left(g_{f i} v_{r, i}-b_{f i} v_{x, i}\right) \\
-v_{x, f} \cdot \sum_{i \in \Gamma}\left(b_{f i} v_{r, i}+g_{f i} v_{x, i}\right) \\
\mathbf{f}_{q}=-\left(v_{r, f}^{2}+v_{x, f}^{2}\right) \cdot \sum_{i \in \Gamma} b_{f i}+v_{r, f} \cdot \sum_{i \in \Gamma}\left(b_{f i} v_{r, i}+g_{f i} v_{x, i}\right) \\
-v_{x, f} \cdot \sum_{i \in \Gamma}\left(g_{f i} v_{r, i}-b_{f i} v_{x, i}\right)
\end{gathered}
$$

where: $v_{r, f}$ and $v_{x, f}$ are the real and imaginary part of the voltage estimate at the feeder bus, respectively; $\Gamma$ is the set of nodes adjacent to the feeder bus; $v_{r, i}$ and $v_{x, i}$ are the real and imaginary voltage at the adjacent buses $i ; g_{f i}$ and $b_{f i}$ are the real and imaginary components of the series admittance

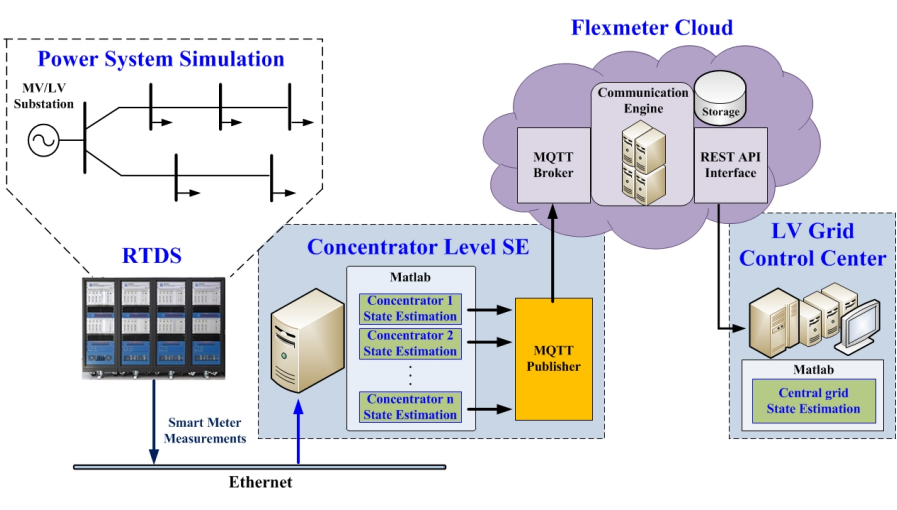

Fig. 4. Real-time simulation platform

in the branch between feeder bus and node $i$. In (8) and (9) the contribution of the shunt capacitive admittance is ignored since in LV grids it is usually negligible.

Using (6), the information about the uncertainty of voltage and power injection at the feeder bus can be extracted, allowing the proper implementation of the WLS approach in the upper level SE.

\section{TESTS AND RESULTS}

\section{A. Real-time test platform}

The smart metering infrastructure and the proposed SE solution have been tested in simulation environment by means of a real-time simulation platform interfaced to the Flexmeter cloud. As shown in Fig. 4, RTDS is used for the real-time simulation of the LV grid. The test network is composed of a MV/LV substation node and two main feeders having five buses in total. Each feeder bus has 15 customers connected to it, which are equally divided among the three phases of the system (Fig. 5). For the simulation purposes, for each customer, a random power profile for the entire day has been generated using the tool presented in [22]. The power flow results of the real-time simulation are assumed as reference values for SE evaluation purposes, and the voltage magnitude and the active and reactive power at each customer node are periodically transmitted to a PC to emulate the smart meter measurements (every minute in the performed simulations). Here, the voltage and power values are perturbed with random noise to simulate the uncertainty of the smart meter. Measurement errors are assumed as independent and Gaussian

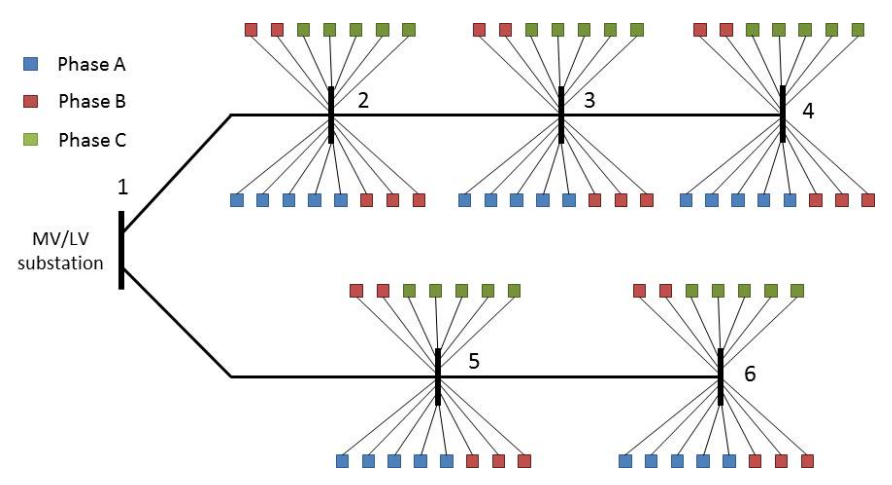

Fig. 5. LV grid topology 
distributed with standard deviation equal to one third of the considered measurement accuracy, which is assumed equal to $1 \%$ and $3 \%$ of the reading value for the voltage and power measurements, respectively.

The smart meter measurements are then acquired by the concentrator level SE. In the simulated scenario, a concentrator is emulated for each feeder bus (nodes from 2 to 6 in Fig. 5). As described in Section III, the concentrator estimators process the measurements for all the customers downstream their feeder bus, and provide as output the voltage and power estimates at the feeder bus together with the corresponding uncertainties. These outputs are then converted to the Flexmeter data format and sent to the cloud through the MQTT publisher. Finally, the concentrator estimates stored in the cloud are periodically required and collected (through the REST/API interface) by the grid level SE. In particular, the central estimator asks for the set of estimates provided by each concentrator in the last predefined period of time (one minute in the simulations). The obtained data are used to perform SE for the 6-bus grid in Fig. 5 and to achieve in this way the corresponding final estimations.

\section{B. Simulation results}

The performed simulations can be divided in two different sets. Firstly, a preliminary off-line validation of the SE architecture has been carried out. Then, the simulation platform described in Section IV-A has been used to evaluate the realtime operation of the software smart metering architecture and of the SE algorithms.

The main goal of the off-line tests has been to perform a preliminary validation of the overall architecture conceived for the smart meter based SE. The performance of the single estimators was already known from previous works (e.g. [19]), thus one of the foci here has been the assessment of the coordination step between concentrator level SE and central grid SE. As described in Section III-A, an important aspect for maximizing the achievable SE accuracy is to verify that the concentrator estimators are capable of correctly evaluating the uncertainty associated to the estimated quantities. In fact, these uncertainties are then used by the upper level SE to assign the weights to its measurement inputs. To this purpose, Monte Carlo (MC) tests have been performed, taking different snapshots of the operating conditions of the grid in different instants of time. Starting from the reference conditions at a given instant of time, each MC simulation has been carried out by running 25000 trials in which different sets of measurements were created by randomly perturbing the reference values of the measured quantities. In this way, it has been possible to compare the statistical uncertainty obtained through the MC simulation to the theoretical one provided by the estimators.

Table I shows as an example the results obtained for the voltage and active/reactive power of phase A of bus 2 during one of the MC simulations. It is worth noting that, since the estimator provides the theoretical uncertainties for each MC trial, different values can be found over the MC simulation. For this reason Table I shows the minimum, average and maximum
TABLE I

STATISTICAL VS THEORETICAL UNCERTAINTY OF CONCENTRATOR SE OUTPUT

\begin{tabular}{|c|c|c|c|c|}
\hline \multirow{2}{*}{$\begin{array}{c}\text { Estimated } \\
\text { quantity }\end{array}$} & Monte Carlo & \multicolumn{3}{|c|}{ Theoretical uncertainty [\%] } \\
\cline { 3 - 5 } & uncertainty [\%] & Minimum & Average & Maximum \\
\hline Voltage & 0.45 & 0.44 & 0.45 & 0.45 \\
\hline Active power & 1.35 & 1.40 & 1.43 & 1.45 \\
\hline Reactive power & 1.35 & 1.40 & 1.43 & 1.45 \\
\hline
\end{tabular}

value of theoretical uncertainty provided by the concentrator estimator during the whole MC simulation. All the results refer to expanded uncertainties with coverage factor equal to three and are expressed in percentage of the estimated quantity. From the obtained results it is possible to observe that a very good matching is achieved for the voltage estimates. As for the powers, small differences were obtained (less than $0.1 \%$ ). The impact of this error was evaluated by comparing the results provided by the central SE when using the weighting matrix designed according to either the theoretical or the MC uncertainty. Performed tests showed negligible differences in the SE results (lower than $0.003 \%$ for the power estimates), thus suggesting that such errors can be considered as acceptable.

MC simulations have been also run to assess the benefits achievable through the central level SE. Found results showed important improvements above all for the voltage estimates. The expanded uncertainty for the voltage estimation at the feeder buses decreases from $0.45 \%$ to $0.2 \%$ when passing from concentrators SE to the central LV grid SE. These results are also in line with the expected voltage uncertainty indicated in [23] according to the number of processed voltage measurements (in the proposed scenario, 5 for each concentrator and, consequently, 25 for the central SE).

Additional tests have been conducted to assess the time performance of the proposed SE architecture. Communication tests have been carried out to estimate the time needed by the concentrator to publish the data through the MQTT protocol into the Flexmeter cloud. Tests have been performed in both Local Area Network (LAN) and Wide Area Network (WAN) environments. To stress these tests, the evaluation was done by emulating a building concentrator publishing data in a payload of about 1 Kbyte every $500 \mathrm{~ms}$. Table II reports the obtained results. As it is possible to observe, when concentrator (publisher), cloud (subscriber) and message broker communicate across Internet, larger delivery times can occur, but in general the average communication time is lower than $100 \mathrm{~ms}$. Table II also shows the computation times (using a $2 \mathrm{GHz}$ quad-core processor with 8GB RAM) obtained for concentrator and LV grid SE (including the time to access the data in the cloud through the REST API). Such results provide a raw assessment of the overall achievable latency, indicating that the proposed architecture is able to provide SE results with a limited delay (within few seconds).

Finally, tests have been carried out to evaluate the realtime operation of the whole SE platform shown in Fig. 4. In this performed simulation, gathering of the smart meter 
TABLE II

TIME PERFORMANCE OF SE ARCHITECTURE COMPONENTS

\begin{tabular}{|c|c|c|c|}
\hline $\begin{array}{c}\text { Tested } \\
\text { component }\end{array}$ & $\begin{array}{c}\min \Delta T \\
{[\mathrm{~ms}]}\end{array}$ & $\begin{array}{c}\max \Delta T \\
{[\mathrm{~ms}]}\end{array}$ & $\begin{array}{c}\text { mean } \Delta T \\
{[\mathrm{~ms}]}\end{array}$ \\
\hline LAN comm. & 2.3 & 13.2 & 3.3 \\
\hline WAN comm. & 79 & 978.5 & 88 \\
\hline Concentrator SE & 8.1 & 18 & 10.1 \\
\hline LV grid SE & 623 & 1294 & 684 \\
\hline
\end{tabular}

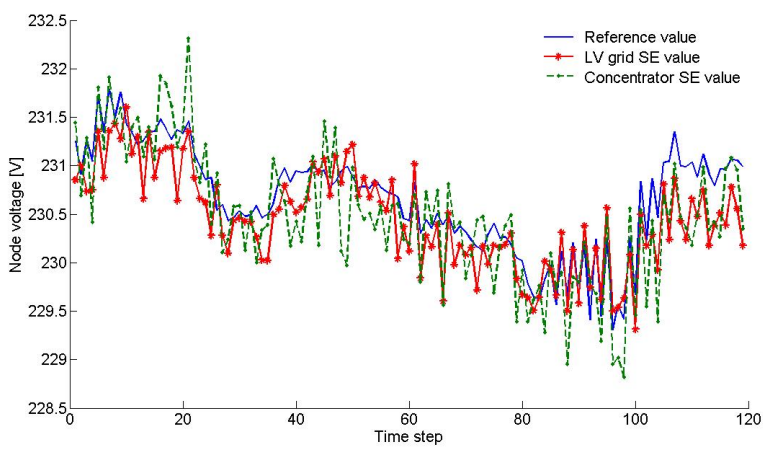

Fig. 6. Estimated vs reference bus voltage at bus 2, phase A

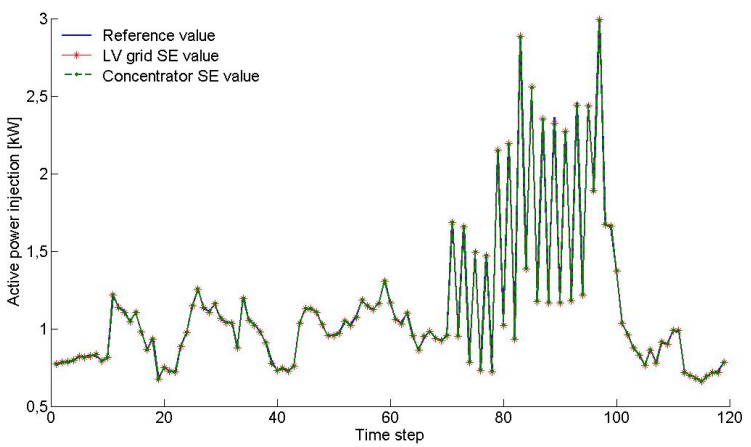

Fig. 7. Estimated vs reference active power injection at bus 2, phase A

measurements and SE are executed with one minute time steps. The presented results refer to a two hours simulation, where the power consumptions of the customers are extracted from the daily profile obtained through the tool in [22]. Fig. 6 shows, as an example, the achieved SE results for the bus voltage estimation at node 2 (phase A) in comparison to the reference true values provided by the real-time simulator. It is possible to see that some small errors are present because of the simulated smart meter uncertainties, but in general the estimator is capable to accurately track the voltage changes in the grid. Fig. 7 shows instead the estimated active power injection for the same bus. Even in this case it is possible to observe that the varying profile of the power over the time can be accurately followed by means of the adopted SE solution.

\section{CONCLUSIONS}

This paper presented a proposal for implementing SE in Low Voltage grids by using smart meter measurements. A cloud-based smart metering architecture has been presented, which allows achieving scalability, flexibility, interoperability, as well as the possibility to obtain access to specific measure- ment data for different service providers. Moreover, a twolevel SE procedure has been proposed to efficiently use smart meter measurements in LV grid SE. The designed solution permits scaling down the SE problem and distributing the computational requirements among different intelligent nodes in the grid.

\section{ACKNOWLEDGEMENTS}

This work was supported by FLEXMETER, which is an EU Horizon 2020 project under grant agreement no. 646568.

\section{REFERENCES}

[1] A. Abur and A. G. Expòsito, Power System State Estimation. Theory and Implementation. Marcel Dekker, New York, 2004

[2] G. Heydt, "The next generation of power distribution systems," IEEE Trans. Smart Grid, vol. 1, no. 3, pp. 225-235, Dec. 2010

[3] D. Della Giustina, M. Pau, P. A. Pegoraro, F. Ponci, and S. Sulis, "Electrical distribution system state estimation: measurement issues and challenges," IEEE Instrumentation Measurement Magazine, vol. 17, no. 6, pp. 36-42, Dec. 2014.

[4] A. Angioni, T. Schlösser, F. Ponci, and A. Monti, "Impact of pseudomeasurements from new power profiles on state estimation in lowvoltage grids," IEEE Transactions on Instrumentation and Measurement, vol. 65, no. 1, pp. 70-77, Jan 2016.

[5] The European Commission, "Commission recommendation on preparations for the roll-out of smart metering systems," Mar. 2012. [Online]. Available: http://eur-lex.europa.eu/legal-content/EN/ ALL/?uri=CELEX:32012H0148

[6] The European Commission, "Benchmarking smart metering deployment in the EU-27 with a focus on electricity," Jul 2014. [Online]. Available: http://eur-lex.europa.eu/legal-content/EN/ TXT/?qid=1403084595595\&uri=COM:2014:356:FIN

[7] FLEXMETER: Flexible smart metering for multiple energy vectors with active prosumers. [Online]. Available: http://flexmeter.polito.it/

[8] IDE4L: Ideal grid for all. [Online]. Available: http://ide4l.eu/

[9] INTREPID: Intelligent systems for energy prosumers buildings at district level. [Online]. Available: http://www.fp7-intrepid.eu/

[10] D. Waeresch, R. Brandalik, W. H. Wellssow, J. Jordan, R. Bischler, and N. Schneider, "Linear state estimation in low voltage grids based on smart meter data," in PowerTech, 2015 IEEE Eindhoven, June 2015, pp. $1-6$.

[11] A. Abdel-Majeed and M. Braun, "Low voltage system state estimation using smart meters," in 2012 47th International Universities Power Engineering Conference (UPEC), Sept 2012, pp. 1-6.

[12] K. Samarakoon, J. Wu, J. Ekanayake, and N. Jenkins, "Use of delayed smart meter measurements for distribution state estimation," in 2011 IEEE Power and Energy Society General Meeting, July 2011, pp. 1-6.

[13] A. Alimardani, F. Therrien, D. Atanackovic, J. Jatskevich, and E. Vaahedi, "Distribution system state estimation based on nonsynchronized smart meters," IEEE Transactions on Smart Grid, vol. 6, no. 6, pp. 2919-2928, Nov. 2015.

[14] E. Patti, A. Acquaviva, and E. Macii, "Enable sensor networks interoperability in smart public spaces through a service oriented approach," in Advances in Sensors and Interfaces (IWASI), 2013 5th IEEE International Workshop on, June 2013, pp. 2-7.

[15] "Message Queue Telemetry Transport (MQTT)," Available: http://mqtt. org/.

[16] P. T. Eugster, P. A. Felber, R. Guerraoui, and A.-M. Kermarrec, "The many faces of publish/subscribe," ACM CSUR, June 2003.

[17] R. T. Fielding, "Architectural styles and the design of network-based software architectures," Irvine: University of California, 2000.

[18] E. Patti, A. L. A. Syrri, M. Jahn, P. Mancarella, A. Acquaviva, and E. Macii, "Distributed software infrastructure for general purpose services in smart grid," IEEE Transactions on Smart Grid, vol. 7, no. 2, pp. 1156-1163, March 2016.

[19] M. Pau, P. A. Pegoraro, and S. Sulis, "Performance of three-phase WLS distribution system state estimation approaches," in Applied Measurements for Power Systems (AMPS), 2015 IEEE International Workshop on, Sept 2015, pp. 138-143.

[20] W. H. Kersting, Distribution System Modeling and Analysis. Boca Raton, 2012. 
[21] M. Asprou, E. Kyriakides, and M. Albu, "The effect of variable weights in a wls state estimator considering instrument transformer uncertainties," IEEE Trans. Instrum. Meas., vol. 63, no. 6, pp. 14841495, Jun. 2014.

[22] C. Molitor, K. Togawa, S. Bolte, and A. Monti, "Load models for home energy system and micro grid simulations," in 2012 3rd IEEE PES Innovative Smart Grid Technologies Europe (ISGT Europe), Oct 2012, pp. 1-6.

[23] M. Pau, P. A. Pegoraro, S. Sulis, and C. Muscas, "Uncertainty sources affecting voltage profile in distribution system state estimation," in Instrumentation and Measurement Technology Conference (I2MTC), 2015 IEEE International, May 2015. 